NOTICE: this is the author's version of a work that was accepted for publication in System and Control Letters. Changes resulting from the publishing process, such as peer review, editing, corrections, structural formatting, and other quality control mechanisms may not be reflected in this document. Changes may have been made to this work since it was submitted for publication. A definitive version was subsequently published in System and Control Letters, Vol. 61, No. 6 (2012). DOI: http://dx.doi.org/10.1016/j.sysconle.2012.02.015 


\title{
The design of nonovershooting and nonundershooting multivariable state feedback tracking controllers
}

\author{
Robert Schmid* and Lorenzo Ntogramatzidis
}

March 28, 2012

\begin{abstract}
We consider the use of linear multivariable feedback control to achieve a nonovershooting and nonundershooting step response. Recently Schmid and Ntogramatzidis [19] introduced a linear state feedback controller design method to avoid overshoot. In this paper we describe conditions under which the design method may be modified to avoid undershoot. The method is applicable to square and non-square systems, minimum and nonminimum phase systems, and also strictly proper and bi-proper systems.
\end{abstract}

Keywords: Tracking control, step response, nonovershooting and nonundershooting linear controllers, MIMO systems.

\section{Introduction}

The problem of designing a linear control law to achieve a nonundershooting or nonovershooting step response for linear time invariant (LTI) systems has been studied for several decades. It is well known that the transient response of an LTI system is related to its zero structure, and numerous studies have reported fundamental performance limitations arising from nonminimum phase (NMP) zeros. A recent comprehensive survey of the impact of system zeros on control system performance is given in [7].

Much of the existing literature on overshoot and undershoot is concerned with singleinput single-output (SISO) systems. Papers offering analytic results on the system overshoot include [14], which considers third-order continuous-time SISO systems, and where necessary and sufficient conditions are given in terms of the closed-loop poles for which the step response is nonovershooting. In [21] it is shown that for a continuous-time SISO system with two nonminimum phase real zeros (right-hand complex plane), the step response must overshoot if the settling time is sufficiently small. Papers offering design

*Author for correspondence. Robert Schmid is with the Department of Electrical and Electronic Engineering, University of Melbourne, Parkville, VIC 3010, Australia. E-mail: rschmid@unimelb.edu.au. Lorenzo Ntogramatzidis is with the Department of Mathematics and Statistics, Curtin University, Perth, WA 6848, Australia. E-mail: L.Ntogramatzidis@curtin.edu.au 
methods include [4], where it is shown how to design a two parameter feedback controller for an LTI continuous-time plant that renders the step response nonovershooting. In [2] an eigenvalue assignment method is proposed to obtain a nonovershooting LTI state feedback controller for continuous-time plants with one nonminimum phase zero. In [3] conditions are given for the existence of a controller to achieve a sign invariant impulse response, and hence also a nonovershooting step response. Corresponding conditions for discrete systems are given in [5].

Analytic results on the system undershoot include [15], where it is shown that an LTI SISO continuous-time system has an undershooting step response if it contains at least one real nonminimum phase zero. A lower bound for the size of the undershoot is also given, and this result is extended in [13] where SISO systems with two real nonminimum phase zeros are considered and a lower bound for the minimum undershoot is given.

Papers simultaneously considering both undershoot and overshoot have generally sought conditions to ensure the step response is monotonic. In [1], the authors give conditions for discrete-time and continuous-time SISO systems to have a nonnegative realization, implying a positive impulse response and hence a monotonic step response. Sufficient conditions for discrete-time SISO systems to have a monotonic step response were also given in [11]. Papers offering design methods for a nonundershooting response have typically aimed at achieving a sign invariant impulse response, such as [3] and [5]. However such an approach is inherently conservative, because a sign invariant impulse response (and hence also a monotonic step response) is not necessary to avoid undershoot or overshoot.

To date there have been few papers offering analysis or design methods for undershoot or overshoot in the step response of multi-input multi-output (MIMO) systems. The paper [8] considered MIMO systems subject to dynamic output feedback, and gave a lower bound on the system undershoot and interaction for systems with at least one real NMP zero. A recent contribution in this area is [19], which employed the classic eigenstructure assignment algorithm of [16], and gave conditions under which a state feedback controller could be obtained to yield a nonovershooting step response for LTI MIMO systems; the design method was applicable to some nonminimum phase systems, and could be applied to both continuous-time and discrete-time systems. In this paper we give conditions under which the design method of [19] may be modified to achieve a step response for MIMO systems that is both nonovershooting and also nonundershooting. It should be noted however that the question of whether a nonundershooting (or nonovershooting) tracking controller can be obtained for an arbitrary LTI system is an open question, even for SISO systems.

The paper is organized as follows. In Section 2 we formulate our tracking problem and formalize the definitions of overshooting and undershooting step response. Section 3 briefly reviews the state feedback controller design method given in [19], and describes conditions under which a gain matrix can be obtained to give a closed-loop system in which only a small number of the system modes appear in each component of the output. Thus each component of the output may be rendered as the sum of just a few real exponentials. 
Section 4 then provides some conditions under which sums of real exponentials have real positive roots. In Section 5 these technical results are incorporated into the design method of [19]. We begin by considering square systems and give conditions under which the design method will yield a state feedback control law that renders the closed-loop system response as nonovershooting, or nonundershooting, or both. Additionally, we give conditions under which the method may be used to yield a monotonic step response. Next we describe briefly how the method for square systems can be modified to accommodate non-square systems. We provide some discussion on the effectiveness of the algorithm, and describe some generic conditions under which the search for a suitable gain matrix is likely to be successful. We summarise the procedure in the form of an algorithm that may be readily coded in MATLAB ${ }^{\circledR}$.

In Section 6 the designed method is applied to two examples. We consider a MIMO system with two inputs/outputs, and two real nonminimum phase zeros. We use our design method to obtain a state feedback law that yields a step response that is monotonic in both outputs. This example shows that several results [6], [15] and [12] describing how the presence of real NMP zeros shape the transient response of a SISO system do not necessarily generalize to the transient response of MIMO systems. In our second example we consider a NMP non-square system, and give a state feedback law that yields a monotonic response from all initial conditions and step references.

\section{Problem Formulation}

Consider the LTI system $\Sigma$ governed by

$$
\Sigma:\left\{\begin{array}{l}
\dot{x}(t)=A x(t)+B u(t), \\
y(t)=C x(t)+D u(t),
\end{array}\right.
$$

where, for all $t \in \mathbb{R}, x(t) \in \mathbb{R}^{n}$ is the state, $u(t) \in \mathbb{R}^{m}$ is the control input, $y(t) \in \mathbb{R}^{p}$ is the output. For time $t<0$, we assume the control input takes a constant value $u_{0}$, which holds the system state at an initial equilibrium $x_{0}$, yielding an output with constant value $y_{0}=C x_{0}+D u_{0}$. Here, $A, B, C$ and $D$ are appropriate dimensional constant matrices. We assume that $B$ has full column rank and $C$ has full row rank. We use $\mathbb{R}^{+}$to denote the set of positive real numbers, and $\mathbb{C}^{-}$to indicate the left hand complex plane. A complex number $\lambda \in \mathbb{C}$ is said to be stable if $\lambda \in \mathbb{C}^{-}$, and a stable matrix is one that has all its eigenvalues contained within $\mathbb{C}^{-}$. In this paper we are concerned with the problem of designing a linear state feedback control law for (1) such that the output $y$ of (1) tracks a step reference $r \in \mathbb{R}^{p}$ with zero steady-state error, and with zero undershoot and overshoot. We adopt the following standard assumption throughout this paper:

Assumption 2.1 System $\Sigma$ is right invertible, stabilisable and has no invariant zeros at the origin.

This assumption ensures that a linear tracking controller can be obtained to track any constant reference $r$ from any given initial condition $x_{0}$ as follows: choose a feedback gain 
matrix $F$ such that $A+B F$ is stable. Two vectors $x_{\mathrm{ss}} \in \mathbb{R}^{n}$ and $u_{\mathrm{ss}} \in \mathbb{R}^{m}$ exist that satisfy

$$
\begin{aligned}
& 0=A x_{\mathrm{ss}}+B u_{\mathrm{ss}} \\
& r=C x_{\mathrm{ss}}+D u_{\mathrm{ss}}
\end{aligned}
$$

for any $r \in \mathbb{R}^{p}$. Application of the control input

$$
u(t)=\left\{\begin{array}{cc}
u_{0}, & t<0 \\
F\left(x(t)-x_{\mathrm{ss}}\right)+u_{\mathrm{ss}}, & t \geq 0
\end{array}\right.
$$

to $\Sigma$ yields the output

$$
y(t)=\left\{\begin{array}{cc}
y_{0}, & t<0 \\
C x(t)+D u(t), & t \geq 0
\end{array}\right.
$$

Employing the change of variable $\xi:=x-x_{\mathrm{ss}}$, we obtain the closed loop homogeneous system

$$
\Sigma_{\text {hom }}:\left\{\begin{array}{l}
\dot{\xi}(t)=(A+B F) \xi(t), \\
y(t)=(C+D F) \xi(t)+r .
\end{array}\right.
$$

Since $A+B F$ is stable, $x$ converges to $x_{\mathrm{ss}}, u$ converges to $u_{\mathrm{ss}}$, and $y$ converges to $r$ as $t$ goes to infinity. For a SISO system, overshoot means that the output exceeds the target $r \in \mathbb{R}$, while undershoot means that the output moves further away from the target than its initial distance. Thus, if $y_{0}<r$, undershoot occurs if the response $y(t)$ takes values less than or equal to $y_{0}$, while overshoot occurs if $y(t)$ takes values greater than or equal to $r$. If $y_{0}>r$, undershoot occurs if the response $y(t)$ takes values greater than or equal to $y_{0}$, and overshoot occurs if $y(t)$ takes values less than or equal to $r$.

It is also helpful to understand the system response in terms of the tracking error $\epsilon(t)=r-y(t)$. At the initial equilibrium $\left(u_{0}, x_{0}, y_{0}\right)$, we have $\epsilon_{0}=r-y_{0}$. Thus overshoot corresponds to the tracking error crossing the time axis. For $y_{0}<r$, undershoot corresponds to the tracking error taking values greater than or equal to its initial value $\epsilon_{0}$, while for $y_{0}>r$ it corresponds to the error taking values smaller than its initial value $\epsilon_{0}$.

Figure 1 shows some examples of step responses $y(t)$ and the corresponding tracking errors $\epsilon(t)=r-y(t)$ with $y_{0}<r$. Notice that $y_{1}$, and $y_{2}$ are all overshooting and nonundershooting step responses, while $y_{3}$ and $y_{4}$ are all nonovershooting but undershooting.

Figure 2 shows some examples of step responses and tracking errors with $y_{0}>r$. Again, $y_{1}$, and $y_{2}$ are examples of overshooting and nonundershooting responses, while $y_{3}$ and $y_{4}$ are nonovershooting and undershooting. Intuitively, we may say that overshoot or undershoot occur when the output curve goes outside the 'train tracks' formed by the horizontal lines $y=y_{0}$ and $y=r$.

Note that for bi-proper systems (i.e., with $D \neq 0$ ), the instantaneous change in the control input at $t=0$ may lead to instantaneous changes in the output at $t=0$. Thus in general for $D \neq 0$, we have $y_{0} \neq y(0)$. For example, in Figure 1 we can see that the responses $y_{1}, y_{2}$ and $y_{3}$ are all characterized by an instantaneous transition from $y_{0}$ to 

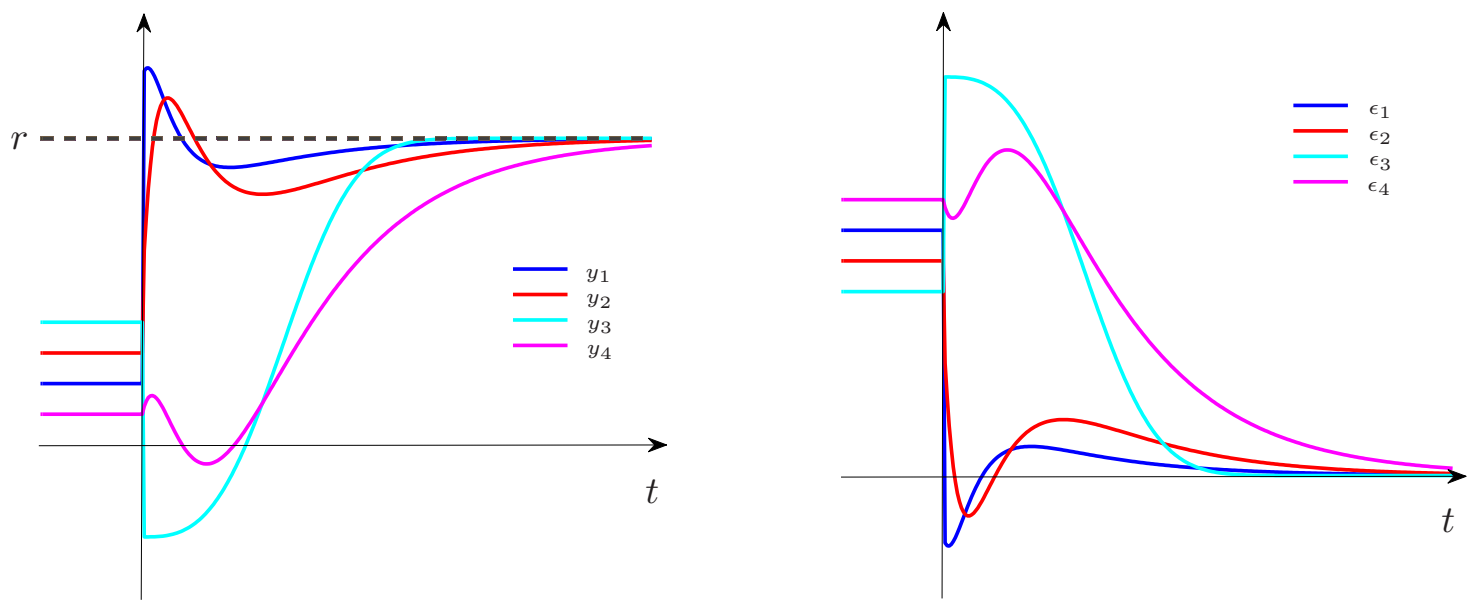

Figure 1: Possible step response curves and corresponding tracking errors with $y_{0}<r$.
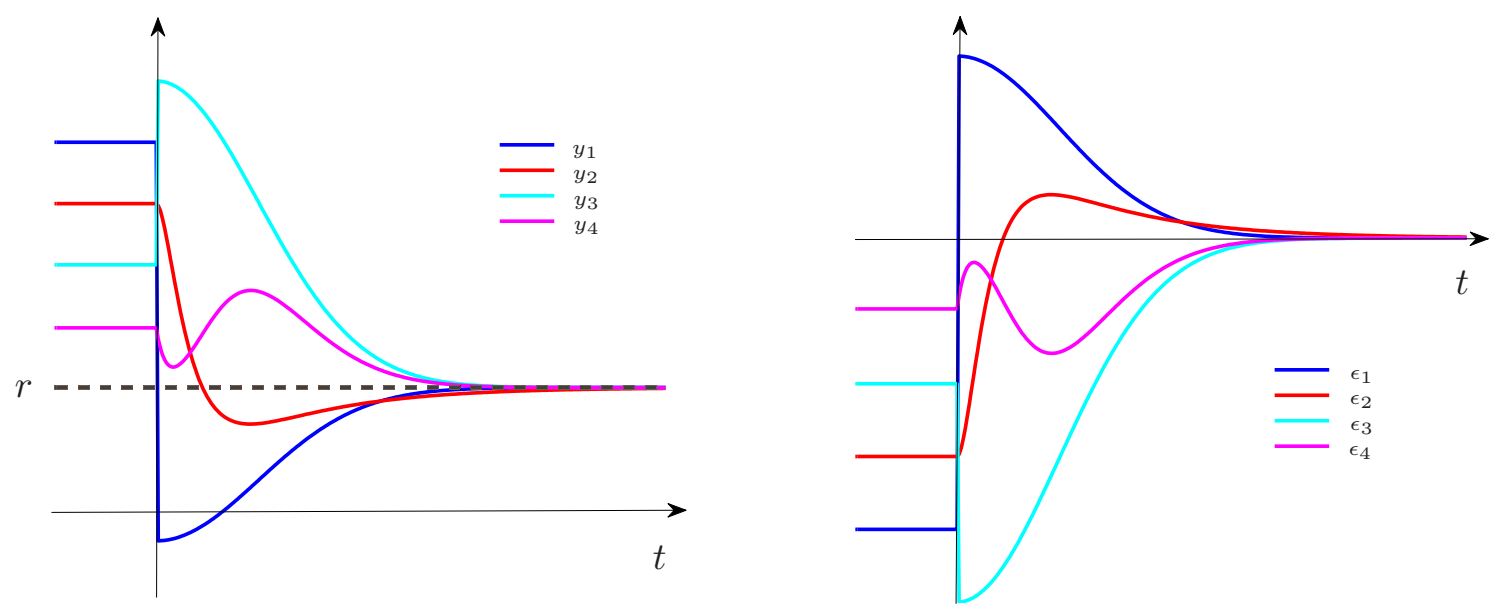

Figure 2: Possible step response curves and corresponding tracking errors with $y_{0}>r$.

$y(0)$ at $t=0$. For $y_{0}<r$, we must have $y_{0} \leq y(0)<r$, else overshoot or undershoot occur immediately. This is the case of $y_{1}$ and $y_{3}$ in Figure 1: indeed, $y_{1}(0)>r$, so that we have instantaneous overshoot, and $y_{3}(0)<y_{0}$, so that we have instantaneous undershoot.

Conversely, for $y_{0}>r$, we must have $r<y(0) \leq y_{0}$. This does not hold for $y_{1}$ and $y_{3}$ in Figure 2: we have $y_{1}(0)<r$, implying instantaneous overshoot, and $y_{3}(0)>y_{0}$, implying instantaneous undershoot.

To diagnose cases where overshoot and undershoot may occur instantaneously due to discontinuities in the output arising from $D \neq 0$, we may write the error $\epsilon(0)=\mu \epsilon_{0}$, for some $\mu \in \mathbb{R}$. Then $\mu \leq 0$ corresponds to instantaneous overshoot, since it leads to an instantaneous change of sign in the error function at $t=0$. If $0<\mu \leq 1$, then 
overshoot occurs if there exists $\bar{t} \in \mathbb{R}^{+}$such that $\epsilon_{0} \epsilon(\bar{t})<0$. Lastly $\mu>1$ corresponds to instantaneous undershoot, since the inequalities $\epsilon(0)>\epsilon_{0}$ and $\epsilon(0)<\epsilon_{0}$ in the cases $y_{0}<r$ and $y_{0}>r$, respectively, can be written as $\epsilon(0)=\mu \epsilon_{0}$ with $\mu>1$. For strictly proper systems we will always have $\mu=1$.

A key technical task of this paper is to establish simple tests for overshoot and undershoot, in terms of the error function. We have noted that overshoot corresponds to the error function crossing the time axis. Since the outputs of system $\Sigma$ are continuous functions of time, this means the error function will have a root, i.e if overshoot occurs, there exists $\bar{t} \in \mathbb{R}^{+}$such that $\epsilon(\bar{t})=0$.

Note however that it is possible for the error function to have a root without changing sign, if the root is also a turning point. In this very special case the output reaches the constant target exactly, but then moves back from it without exceeding it. Thus, to properly define overshoot in terms of roots of the error function, we should say that overshoot occurs when the error term has at least one root that is not also a turning point. This would however make the task of testing for overshoot very difficult, since it would require locating the roots and testing them until at least one of them was found to not also be a turning point. Instead, we adopt the simpler approach of defining overshoot to occur if the error function has a root, whether or not the root is also a turning point. This is a slightly more conservative definition of overshoot, since it declares the output to be overshooting in some cases where, strictly speaking, it is not. Since the aim of our design method is to avoid overshoot, and given the exceptional rarity of such situations, this mild conservatism is acceptable for our purposes.

An analogous situation arises with respect to undershoot. Strictly speaking, undershoot occurs if there exists $\bar{t} \in \mathbb{R}^{+}$such that $\epsilon(\bar{t})>\epsilon_{0}$. Again, this situation is very difficult to test for; we shall instead test for the condition $\epsilon(\bar{t})=\epsilon_{0}$, which again involves a mild conservatism. The following definition formalizes the above discussion.

Definition 2.1 Assume the initial control input, state and output of the system $\Sigma$ are at an equilibrium $\left(u_{0}, x_{0}, y_{0}\right)$. Let $r \in \mathbb{R}^{p}$ be a step reference and let $\epsilon=r-y$ be the tracking error obtained when the control input u given in (4) is applied to $\Sigma$. Define $\epsilon_{0}=r-y_{0}$, and for each component $k \in\{1, \ldots, p\}$, let $\epsilon_{0, k}$ denote the $k$-th component of $\epsilon_{0}$. Then

(i) the system $\Sigma$ has an overshooting response in the output component $y_{k}(t)(k \in$ $\{1, \ldots, p\})$ for a given $r \in \mathbb{R}^{p}$ from the initial condition $\left(u_{0}, x_{0}, y_{0}\right)$ if either $(a) \epsilon_{k}(0)=$ $\mu_{k} \epsilon_{0, k}$, for some $\mu_{k}<0$, or (b) the tracking error $\epsilon_{k}$ has a positive real root, i.e., there exists a $\bar{t} \in \mathbb{R}^{+}$such that $\epsilon_{k}(\bar{t})=0$, or $(c) \epsilon_{k}(0)=0$, and there exists $\bar{t} \in \mathbb{R}^{+}$such that $\epsilon_{0, k} \epsilon_{k}(\bar{t})<0$;

(ii) the system $\Sigma$ has an undershooting response in the output component $y_{k}(t)(k \in$ $\{1, \ldots, p\})$ for a given $r \in \mathbb{R}^{p}$ from the initial condition $\left(u_{0}, x_{0}, y_{0}\right)$ if either $(a) \epsilon_{k}(0)=$ $\mu_{k} \epsilon_{0, k}$, for some $\mu_{k}>1$, or (b) there is a $\bar{t} \in \mathbb{R}^{+}$such that $\epsilon_{k}(\bar{t})=\epsilon_{0, k}$;

(iii) if $\Sigma$ is nonovershooting (respectively, nonundershooting, monotonic) in all output components for a given $r \in \mathbb{R}^{p}$, then we say $\Sigma$ has a nonovershooting (respectively, nonundershooting, monotonic) response for the specified $\left(u_{0}, x_{0}, y_{0}\right)$ and $r$. 
(iv) if $\Sigma$ is nonovershooting (respectively, nonundershooting, monotonic) in all output components for a given $r \in \mathbb{R}^{p}$ from all initial equilibria $\left(u_{0}, x_{0}, y_{0}\right)$, then we say $\Sigma$ has a globally nonovershooting (respectively, globally nonundershooting, globally monotonic) response for this $r$.

Our aim in this paper is to design, for any given initial equilibrium $\left(u_{0}, x_{0}, y_{0}\right)$ and target reference $r$, a gain matrix $F$ such that the control law (4) moves the system to a final equilibrium $\left(u_{s s}, x_{s s}, r\right)$ without overshoot or undershoot in the transient response.

\section{$3 \quad$ Feedback controller design method}

In [19] a linear state feedback design method was introduced to yield a nonovershooting step response. The main idea of the method was to employ the classic result on eigenstructure assignment given by B.C. Moore in [16] to constrain the output $y(t)$ in such a way that each component of the error term $\epsilon(t)$ contained only a small number of the closed-loop modes (poles). Here we summarize the design method, and present some results that describe the form of the error term. In Section 5 we consider how the design method may be employed to yield a closed loop response that is both nonovershooting and nonundershooting. A key result is the following eigenstructure lemma from [19], which is itself an adaptation of Moore's algorithm.

Lemma 3.1 [19] Let $\mathcal{L}=\left\{\lambda_{1}, \ldots, \lambda_{n}\right\}$ be a self-conjugate set of $n$ distinct complex numbers. Let $\mathcal{S}=\left\{s_{1}, \ldots, s_{n}\right\}$ be a set of $n$ (not necessarily distinct) vectors in $\mathbb{R}^{p}$. Assume that, for each $i \in\{1, \ldots, n\}$, the matrix equation

$$
\left[\begin{array}{cc}
A-\lambda_{i} I & B \\
C & D
\end{array}\right]\left[\begin{array}{c}
v_{i} \\
w_{i}
\end{array}\right]=\left[\begin{array}{c}
0 \\
s_{i}
\end{array}\right]
$$

has solutions sets $\mathcal{V}=\left\{v_{1}, \ldots, v_{n}\right\} \subset \mathbb{C}^{n}$ and $\mathcal{W}=\left\{w_{1}, \ldots, w_{n}\right\} \subset \mathbb{C}^{m}$. Then, provided $\mathcal{V}$ is linearly independent, a unique real feedback matrix $F$ exists such that, for all $i \in$ $\{1, \ldots, n\}$,

$$
\begin{aligned}
& (A+B F) v_{i}=\lambda_{i} v_{i}, \\
& (C+D F) v_{i}=s_{i} .
\end{aligned}
$$

Remark 3.1 For square systems $(p=m)$, if $s_{i}=0$, then (7) is solvable for non-zero $v_{i}$ if and only if $\lambda_{i}$ is a zero of $\Sigma$. For arbitrary non-zero $s_{i} \in \mathbb{R}^{p},(7)$ is solvable if and only if $\lambda_{i}$ is real, and not a zero of $\Sigma$, by right invertibility. Assuming $\mathcal{L}$ and $\mathcal{S}$ are such that the solution sets $\mathcal{V}$ and $\mathcal{W}$ do exist, then Moore's algorithm (Proposition 1 of [16]) can be used to construct $F$ : Obtain real vectors $\hat{\mathcal{V}}=\left\{\hat{v}_{1}, \ldots, \hat{v}_{n}\right\} \subset \mathbb{R}^{n}$ and $\hat{\mathcal{W}}=\left\{\hat{w}_{1}, \ldots, \hat{w}_{n}\right\} \subset \mathbb{R}^{m}$ as follows: for each $i \in\{1, \ldots, n\}$,

(i) If $\lambda_{i} \in \mathbb{R}$, define $\hat{v}_{i}=v_{i}$ and $\hat{w}_{i}=w_{i}$. 
(ii) If $\lambda_{i}, \lambda_{i+1} \in \mathbb{C}$ are a complex conjugate pair, define $\hat{v}_{i}=\operatorname{Re}\left(v_{i}\right), \hat{v}_{i+1}=\operatorname{Im}\left(v_{i}\right)$, $\hat{w}_{i}=\operatorname{Re}\left(w_{i}\right), \hat{w}_{i+1}=\operatorname{Im}\left(w_{i}\right)$, where $\operatorname{Re}$ and $\operatorname{Im}$ denote the real and imaginary components of a complex vector, respectively.

(iii) Define $\hat{V}=\left[\hat{v}_{1} \ldots \hat{v}_{n}\right]$ and $\hat{W}=\left[\hat{w}_{1} \ldots \hat{w}_{n}\right]$ and solve

$$
F=\hat{W} \hat{V}^{-1}
$$

\subsection{Systems with at least $n-p$ minimum phase zeros}

The first design method applies to systems that satisfy

Assumption 3.1 System $\Sigma$ is square $(m=p)$ has at least $n-p$ distinct invariant zeros in $\mathbb{C}^{-}$.

Let $\mathcal{L}=\left\{\lambda_{1}, \ldots, \lambda_{n}\right\} \subset \mathbb{C}^{-}$denote the set of distinct stable closed loop eigenvalues of $A+B F$ to be chosen. Let $\left\{z_{1}, z_{2}, \ldots, z_{n-p}\right\} \subset \mathbb{C}^{-}$be freely chosen from among the distinct minimum phase invariant zeros of $\Sigma$. Then, we choose $\lambda_{i}=z_{i}$ for $i \in\{1, \ldots, n-p\}$; these modes are stable as all $z_{i}$ lie in $\mathbb{C}^{-}$. For $i \in\{n-p+1, \ldots, n\}$, the $\lambda_{i}$ may be freely chosen to be any real distinct stable modes not coincident with invariant zeros of $\Sigma$. Let $\left\{e_{1}, \ldots, e_{p}\right\}$ be the canonical basis of $\mathbb{R}^{p}$, and let $\mathcal{S}=\left\{s_{1}, \ldots, s_{n}\right\} \subset \mathbb{R}^{p}$ be such that

$$
s_{i}=\left\{\begin{array}{cl}
0 & \text { for } i \in\{1, \ldots, n-p\} \\
e_{1} & \text { for } i=n-p+1 \\
\vdots & \\
e_{p} & \text { for } i=n
\end{array}\right.
$$

Observe from Remark 3.1 that $\mathcal{L}$ and $\mathcal{S}$ are such that solution sets $\mathcal{V}=\left\{v_{1}, \ldots, v_{n}\right\} \subset \mathbb{C}^{n}$ and $\mathcal{W}=\left\{w_{1}, \ldots, w_{n}\right\} \subset \mathbb{C}^{p}$ for $(7)$ do exist. Provided $\mathcal{V}$ is linearly independent, by Lemma 3.1, Moore's algorithm yields $F$ such that $A+B F$ has eigenstructure given by $\mathcal{L}$ and $\mathcal{V}$, respectively. Since $w_{i}=F v_{i}$, the vectors in $\mathcal{V}$ satisfy

$$
\begin{aligned}
(A+B F) v_{i} & =\lambda_{i} v_{i}, \quad i \in\{1, \ldots, n\}, \\
(C+D F) v_{i} & = \begin{cases}0 & i \in\{1, \ldots, n-p\}, \\
e_{i-(n-p)} & i \in\{n-p+1, \ldots, n\} .\end{cases}
\end{aligned}
$$

Now introduce the new state coordinate $\xi:=x-x_{\mathrm{ss}}$. As $\mathcal{V}$ is linearly independent, the ma$\operatorname{trix} V:=\left[\begin{array}{llll}v_{1} & v_{2} & \ldots & v_{n}\end{array}\right]$ is invertible. Introduce $\xi_{0}=x_{0}-x_{s s}$ and $\alpha:=\left[\begin{array}{llll}\alpha_{1} & \alpha_{2} & \ldots & \alpha_{n}\end{array}\right]^{\top}=$ $V^{-1} \xi_{0}$. Theorem 3.1 describes the form of the error term:

Theorem 3.1 [19] Assume $\Sigma$ satisfies Assumption 3.1. Let $\mathcal{L}$ be chosen as above, let $F$ be obtained from Moore's algorithm, let $r \in \mathbb{R}^{p}$ be any step reference, and let $\left(u_{0}, x_{0}, y_{0}\right)$ be the initial equilibrium. Then, the error term $\epsilon$ obtained from applying $u$ in (4) to $\Sigma$ has the form

$$
\epsilon(t)=\left[\begin{array}{c}
\alpha_{n-p+1} e^{\lambda_{n-p+1} t} \\
\vdots \\
\alpha_{n} e^{\lambda_{n} t}
\end{array}\right] .
$$

We observe that each of the $p$ components of $\epsilon$ contain exactly one mode. 


\subsection{Systems with fewer than $n-p$ minimum phase zeros}

Next we consider how the design method given in Section 3.1 can be extended to systems with fewer than $n-p$ zeros. Let us denote by $z_{\min }$ the number of minimum phase zeros of $\Sigma$ and we can then write

$$
z_{\text {min }}=n-l p+q
$$

for some integers $l$ and $q$, with $0 \leq q<p$. Firstly let us suppose for simplicity that $q=0$ and we have exactly $n-l p$ minimum phase zeros. The key idea is to channel $l$ modes per output component. Choose $\mathcal{L}=\left\{\lambda_{1}, \ldots, \lambda_{n}\right\}$ with $\lambda_{i}=z_{i}$ for $i \in\{1, \ldots, n-l p\}$, and for $i \in\{n-l p+1, \ldots, n\}$ the $\lambda_{i}$ may be freely chosen to be any distinct real stable modes that are not coincident with invariant zeros of $\Sigma$. Next let $\mathcal{S}=\left\{s_{1}, \ldots, s_{n}\right\} \subset \mathbb{R}^{p}$ be such that

$$
s_{i}=\left\{\begin{array}{cl}
0 & \text { for } i \in\{1, \ldots, n-l p\} ; \\
e_{1} & \text { for } i \in\{n-l p+1, \ldots, n-l(p-1)\} ; \\
e_{2} & \text { for } i \in\{n-l(p-1)+1, \ldots, n-l(p-2)\} \\
\vdots & \\
e_{p} & \text { for } i \in\{n-p+1, \ldots, n\} .
\end{array}\right.
$$

As before, $\mathcal{L}$ and $\mathcal{S}$ are such that solution sets $\mathcal{V}=\left\{v_{1}, \ldots, v_{n}\right\} \subset \mathbb{C}^{n}$ and $\mathcal{W}=$ $\left\{w_{1}, \ldots, w_{n}\right\} \subset \mathbb{C}^{p}$ for $(7)$ do exist. Provided $\mathcal{V}$ is linearly independent, Moore's algorithm yields $F$ such that $A+B F$ has distinct eigenvalues and eigenvectors given by $\mathcal{L}$ and $\mathcal{V}$, respectively. The following notation allows us to succinctly state our theorem for the form of the tracking error term in this case.

Notation 3.1 For each $k \in\{1, \ldots, p\}$, we let

(i) $v_{k, 1}, v_{k, 2}, \ldots, v_{k, l}$ denote the eigenvectors in $\mathcal{V}$ associated with canonical basis vector $e_{k}$ in (16), and let $\lambda_{k, 1}, \lambda_{k, 2}, \ldots \lambda_{k, l}$ be the corresponding real eigenvalues in $\mathcal{L}$, ordered such that $\lambda_{k, 1}<\lambda_{k, 2}<\cdots<\lambda_{k, l}$ in each case;

(ii) For any $x \in \mathbb{R}^{n}$, introduce the new state coordinates $\xi=x-x_{\mathrm{ss}}$ and let $\xi_{0}=\xi(0)$.

Let $V$ be the matrix formed by the columns of $\mathcal{V}$, and let $\alpha:=V^{-1} \xi_{0}$ be the coordinate vector of $\xi_{0}$ in terms of $\mathcal{V}$. Then define

$$
\alpha=\left[\begin{array}{llllllllll}
\alpha_{1} & \ldots & \alpha_{n-l p} & \alpha_{1,1} & \ldots & \alpha_{1, l} & \ldots & \alpha_{p, 1} & \ldots & \alpha_{p, l}
\end{array}\right]^{\top} .
$$

Theorem 3.2 [19] Assume $\Sigma$ is square and has exactly $n-l p$ minimum phase zeros. Let $\mathcal{L}$ be chosen as above, let $F$ be obtained from Moore's algorithm, let $r \in \mathbb{R}^{p}$ be any step reference, and let $x_{0} \in \mathbb{R}^{n}$ be any initial condition. Then $\epsilon_{k}$, the $k$-th component of the error term $\epsilon$ obtained from applying $u$ in (4) to $\Sigma$ has the form

$$
\epsilon_{k}(t)=\alpha_{k, 1} e^{\lambda_{k, 1} t}+\alpha_{k, 2} e^{\lambda_{k, 2} t}+\cdots+\alpha_{k, l} e^{\lambda_{k, l} t}
$$

Remark 3.2 The design method described above employs two rather simple ideas. Firstly, the available minimum phase invariant zeros are used to render the corresponding modes invisible in the tracking error via pole/zero cancelation. The remaining modes are distributed evenly into the $p$ components of the tracking error. Thus, in Theorem $3.1, n-p$ 
modes are canceled and the remaining $p$ modes are allocated with one mode per component. In Theorem 3.2, $n-l p$ modes are canceled and the remaining $l p$ modes are distributed with $l$ modes per component.

Finally if $q>0$ in (15), then we have $n-l p+q$ minimum phase zeros, and the additional minimum phase zeros may be employed by modifying the targets $s_{i}$ in (16) so that $s_{i}=0$ for $i \in\{1, \ldots, n-l p+q\}$. Then we would choose $q$ of the outputs to have only $l-1$ modes allocated into them; the $q$ canonical basis vectors corresponding to those outputs need be associated with only $l-1$ modes each. The remaining $p-q$ outputs would have $l$ modes allocated into them as in (16).

Remark 3.3 Let us use $z_{\text {total }}$ to denote the total number of invariant zeros of $\Sigma$. For square bi-proper $\Sigma$, [17] gives the equation $z_{\text {total }}=n-p+\operatorname{rank} D$. Hence, if $D$ is of full rank, system $\Sigma$ has $n$ zeros. Alternatively, if $\Sigma$ is strictly proper $(D=0)$ and $C B$ is nonsingular, then $z_{\text {total }}=n-p$ [17]. It should be noted that Theorems 3.1 and 3.2 above are only concerned with $z_{\text {min }}$, the number of minimum phase zeros. The remaining system zeros may be of nonminimum phase and do not impact upon the design method.

We see that this eigenstructure based method for designing the feedback matrix $F$ gives us an error term that has a rather simple form; each component contains at most $l$ distinct exponential terms. Other MIMO pole placement methods, for example those in [9], that do not allow the designer to simultaneously select the eigenvectors lead to an error term in which all $n$ modes contribute in all $p$ output components. In Section 5 we introduce methods of exploiting the simplified form of the error term to obtain a suitable gain matrix $F$ that yields a nonovershooting and nonundershooting step response.

\section{Conditions implying Overshoot and Undershoot}

In this section we consider functions composed of sums of real exponential functions, and obtain some technical conditions under which these functions contain a real positive root. When applied to the system tracking error $\epsilon$, such roots correspond to overshoot or undershoot. When applied to the derivative of the tracking error $\dot{\epsilon}$, such roots correspond to the existence of local extrema, which imply a non-monotonic step response. The following result from the classic paper [10] provides information on the number of real roots of such functions in terms of the variations of the signs of their coefficients.

Notation 4.1 Let $l \in \mathbb{N}$, let $\left\{\lambda_{1}, \ldots, \lambda_{l}\right\}$ and $\left\{\alpha_{1}, \ldots, \alpha_{l}\right\}$ be sets of real numbers with $\lambda_{1}<\lambda_{2}<\cdots<\lambda_{l}$. Define $f: \mathbb{R} \rightarrow \mathbb{R}$ as

$$
f(t)=\alpha_{1} e^{\lambda_{1} t}+\alpha_{2} e^{\lambda_{2} t}+\cdots+\alpha_{l} e^{\lambda_{l} t} .
$$

We let $C\left\{\alpha_{1}, \ldots, \alpha_{l}\right\}$ denote the number of changes in the signs in the sequence of coefficients $\left\{\alpha_{1}, \alpha_{2}, \ldots, \alpha_{l}\right\}$, and for any interval $\mathcal{I} \subseteq \mathbb{R}, Z_{\mathcal{I}}^{f}$ denotes the number of real roots of $f$ in $\mathcal{I}$.

Let us also introduce $p_{1}=\alpha_{1}, p_{2}=\alpha_{1}+\alpha_{2}, p_{3}=\alpha_{1}+\alpha_{2}+\alpha_{3}, \ldots, p_{l}=\alpha_{1}+\cdots+\alpha_{l}$ 
and $q_{1}=p_{1}\left(\lambda_{1}-\lambda_{2}\right), q_{2}=q_{1}+p_{2}\left(\lambda_{2}-\lambda_{3}\right), \ldots, q_{l-1}=q_{l-2}+p_{l-1}\left(\lambda_{l-1}-\lambda_{l}\right), q_{l}=p_{l}$. Also introduce $r_{1}=\alpha_{l}, r_{2}=\alpha_{l}+\alpha_{l-1}, r_{3}=\alpha_{l}+\alpha_{l-1}+\alpha_{l-2}, \ldots, r_{l}=\alpha_{l}+\cdots+\alpha_{1}$ and $s_{1}=r_{1}\left(\lambda_{l}-\lambda_{l-1}\right), s_{2}=s_{1}+r_{2}\left(\lambda_{l-1}-\lambda_{l-2}\right), \ldots, s_{l-1}=s_{l-2}+r_{l-1}\left(\lambda_{2}-\lambda_{1}\right), s_{l}=r_{l}$.

Lemma 4.1 [10, Section 24]. Let $f$ be defined as in (19). Then

$$
\begin{aligned}
Z_{(0,1)}^{f} & \leq C\left\{q_{1}, \ldots, q_{l}\right\}, \\
Z_{[1, \infty)}^{f} & \leq C\left\{s_{1}, \ldots, s_{l}\right\} .
\end{aligned}
$$

Our first lemma provides conditions under which the sum of two or three real exponentials contains a real root, which corresponds to overshoot in the step response.

Lemma 4.2 [19] Let $\lambda_{1}<\lambda_{2}<\lambda_{3}<0$, and for any real constants $\left\{\alpha_{1}, \alpha_{2}, \alpha_{3}\right\}$ with $\alpha_{3} \neq 0$, define

$$
f(t)=\alpha_{1} e^{\lambda_{1} t}+\alpha_{2} e^{\lambda_{2} t}+\alpha_{3} e^{\lambda_{3} t}
$$

There exists $\bar{t} \in \mathbb{R}^{+}$such that $f(\bar{t})=0$ if and only if one of the following conditions hold:

I. $C\left\{\alpha_{1}, \alpha_{2}, \alpha_{3}\right\}=1$ and $\left(\alpha_{1}+\alpha_{2}+\alpha_{3}\right) \alpha_{3}<0$;

II. $C\left\{\alpha_{1}, \alpha_{2}, \alpha_{3}\right\}=2$ and $\left(\alpha_{1}+\alpha_{2}+\alpha_{3}\right) \alpha_{3} \geq 0$;

III. $C\left\{\alpha_{1}, \alpha_{2}, \alpha_{3}\right\}=2,\left(\alpha_{1}+\alpha_{2}+\alpha_{3}\right) \alpha_{3}<0, t^{*}>0$ and $\left|g\left(t^{*}\right)\right| \geq\left|\alpha_{1}+\alpha_{2}+\alpha_{3}\right|$, where

$$
\begin{aligned}
t^{*} & =\frac{1}{\lambda_{3}-\lambda_{1}} \ln \left(\frac{\alpha_{1}\left(\lambda_{2}-\lambda_{1}\right)}{\alpha_{3}\left(\lambda_{3}-\lambda_{2}\right)}\right) \\
g(t) & =\alpha_{1}\left(1-e^{\left(\lambda_{1}-\lambda_{2}\right) t}\right)+\alpha_{3}\left(1-e^{\left(\lambda_{3}-\lambda_{2}\right) t}\right) .
\end{aligned}
$$

Proof: The proof appears in [19]; here we have streamlined the presentation by adopting the Notation 4.1.

Note that if $f$ consists of the sum of only two exponentials then we may employ Lemma 4.2 with $\alpha_{1}=0$; in this case only condition $I$. of Lemma 4.2 is relevant.

The next lemma gives a necessary and sufficient condition for the sum of two real exponentials to take on a specified non-zero value, which corresponds to undershoot.

Lemma 4.3 Let $\lambda_{1}<\lambda_{2}<0$, and for any real nonzero constants $\left\{\alpha_{1}, \alpha_{2}\right\}$, define

$$
f(t)=\alpha_{1} e^{\lambda_{1} t}+\alpha_{2} e^{\lambda_{2} t} .
$$

Let $\beta=\frac{1}{\mu}\left(\alpha_{1}+\alpha_{2}\right)$ for some $0<\mu \leq 1$. Then there exists $\bar{t} \in \mathbb{R}^{+}$such that $f(\bar{t})=\beta$ if and only if $t^{*} \in \mathbb{R}^{+}$and $f\left(t^{*}\right) \beta \geq \beta^{2}$, where

$$
t^{*}=\frac{1}{\lambda_{2}-\lambda_{1}} \ln \left(\frac{-\lambda_{1} \alpha_{1}}{\lambda_{2} \alpha_{2}}\right) .
$$

Proof: Since $\lambda_{1}<\lambda_{2}<0$, function $f(t)$ goes to 0 as $t \rightarrow \infty$. Since $\beta=f(0) / \mu$, in the case $f(0)=\alpha_{1}+\alpha_{2}>0$, a $\bar{t}$ exists for which $f(\bar{t})=\beta$ if and only if $f(t)$ has a maximum whose value exceeds $\beta$, i.e., there exists $t^{*}>0$ such that $\dot{f}\left(t^{*}\right)=0$ and $f\left(t^{*}\right) \geq \beta$. If $f(0)<0$, such $\bar{t}$ exists if and only if $f(t)$ has a minimum whose value is more negative 
than $\beta$, i.e., a $t^{*}>0$ exists such that $\dot{f}\left(t^{*}\right)=0$ and $f\left(t^{*}\right) \leq \beta$. Setting $\dot{f}\left(t^{*}\right)=0$ yields $t^{*}$ given by (24). If $t^{*} \notin \mathbb{R}^{+}$, then $f$ is monotonic on $\mathbb{R}^{+}$and takes its extreme value at $t=0$, implying $|f(t)|<\left|\alpha_{1}+\alpha_{2}\right| \leq|\beta|$ for all $t \in \mathbb{R}^{+}$. If $t^{*} \in \mathbb{R}^{+}$, then $f$ takes its extreme value at $t=t^{*}$. As such, $\bar{t} \in \mathbb{R}^{+}$satisfying $f(\bar{t})=\beta$ exists if and only $f\left(t^{*}\right) \beta \geq \beta^{2}$, which captures both the condition $f\left(t^{*}\right) \geq \beta$ when $f(0)>0$ and the condition $f\left(t^{*}\right) \leq \beta$ when $f(0)<0$.

When we apply Lemma 4.3 to test for undershoot, each $\epsilon_{k}(t)$ has the form (23), and we use $\beta_{k}=\epsilon_{0, k}$ for the initial output. Also $\epsilon_{k}(0)=\alpha_{1}+\alpha_{2}$, and we find $\mu_{k} \in \mathbb{R}$ such that $\mu_{k} \epsilon_{0, k}=\epsilon_{k}(0)$. We only need consider $0<\mu \leq 1$ in Lemma 4.3 because $\mu<0$ implies instantaneous overshoot, and $\mu>1$ implies instantaneous undershoot. The following corollary offers a simpler result that can be used for strictly proper systems where no initial discontinuity in the output can occur.

Corollary 4.1 Under the assumptions of Lemma 4.3, if $\mu=1$, then there exists $\bar{t} \in \mathbb{R}^{+}$ such that $f(\bar{t})=\beta$ if and only if and $\left(\alpha_{1}+\alpha_{2}\right)\left(\lambda_{1} \alpha_{1}+\lambda_{2} \alpha_{2}\right)>0$.

Proof: Here $\bar{t} \in \mathbb{R}^{+}$satisfying $f(\bar{t})=\beta$ exists if and only if $f(0) \dot{f}(0)>0$. This holds if and only $\left(\alpha_{1}+\alpha_{2}\right)\left(\lambda_{1} \alpha_{1}+\lambda_{2} \alpha_{2}\right)>0$.

The next lemma gives conditions on when the sum of three real exponentials takes on a specified non-zero value.

Lemma 4.4 Let $\lambda_{1}<\lambda_{2}<\lambda_{3}<0$, and for any nonzero constants $\left\{\alpha_{1}, \alpha_{2}, \alpha_{3}\right\}$, define $\beta=\frac{1}{\mu}\left(\alpha_{1}+\alpha_{2}+\alpha_{3}\right)$ for some $0<\mu \leq 1$. Let

$$
f(t)=\alpha_{1} e^{\lambda_{1} t}+\alpha_{2} e^{\lambda_{2} t}+\alpha_{3} e^{\lambda_{3} t}
$$

and introduce $p_{1}=\alpha_{1}, p_{2}=\alpha_{1}+\alpha_{2}, p_{3}=\alpha_{1}+\alpha_{2}+\alpha_{3}, p_{4}=\alpha_{1}+\alpha_{2}+\alpha_{3}-\beta$ and $q_{1}=p_{1}\left(\lambda_{1}-\lambda_{2}\right), q_{2}=q_{1}+p_{2}\left(\lambda_{2}-\lambda_{3}\right), q_{3}=q_{2}+p_{3}\left(\lambda_{3}\right), q_{4}=p_{4}$. Also introduce $r_{1}=-\beta, r_{2}=\alpha_{3}-\beta, r_{3}=\alpha_{3}+\alpha_{2}-\beta, r_{4}=\alpha_{1}+\alpha_{2}+\alpha_{3}-\beta$ and $s_{1}=r_{1}\left(-\lambda_{3}\right)$, $s_{2}=s_{1}+r_{2}\left(\lambda_{3}-\lambda_{2}\right), s_{3}=s_{2}+r_{1}\left(\lambda_{2}-\lambda_{1}\right), s_{4}=r_{4}$. Then there exists $\bar{t} \in \mathbb{R}^{+}$such that $f(\bar{t})=\beta$ only if at least one of the following conditions hold:

I. $C\left\{q_{1}, q_{2}, q_{3}, q_{4}\right\} \geq 1$;

II. $C\left\{r_{1}, r_{2}, r_{3}, r_{4}\right\} \geq 1$.

Proof: Define $g(t)=f(t)-\beta$. Then $f(\bar{t})=\beta$ for some $\bar{t} \in \mathbb{R}^{+}$if and only $g(\bar{t})=0$. Then we may write $g$ in the form (19) with coefficients $\left\{\alpha_{1}, \alpha_{2}, \alpha_{3},-\beta\right\}$ and exponents $\left\{\lambda_{1}, \lambda_{2}, \lambda_{3}, 0\right\}$. Applying Lemma 4.1 to $g$, we see that $\bar{t}$ exists only if $C\left\{q_{1}, q_{2}, q_{3}, q_{4}\right\} \geq 1$ or $C\left\{r_{1}, r_{2}, r_{3}, r_{4}\right\} \geq 1$.

The generalisation of these lemmas to the sum of any finite number of real exponentials is given by the following:

Lemma 4.5 For some positive integer l, let $\lambda_{1}<\lambda_{2}<\cdots<\lambda_{l}<0$, and for any non-zero constants $\left\{\alpha_{1}, \alpha_{2}, \ldots, \alpha_{l}\right\}$, define

$$
f(t)=\alpha_{1} e^{\lambda_{1} t}+\alpha_{2} e^{\lambda_{2} t}+\cdots+\alpha_{l} e^{\lambda_{l} t} .
$$


(a) Use the sets $\left\{\alpha_{i}: 1 \leq i \leq l\right\}$ and $\left\{\lambda_{i}: 1 \leq i \leq l\right\}$ to obtain sets $\left\{q_{i}: 1 \leq i \leq l\right\}$ and $\left\{s_{i}: 1 \leq i \leq l\right\}$ as in Lemma 4.1. Then there exists $\bar{t} \in \mathbb{R}^{+}$such that $f(\bar{t})=0$ only if $C\left\{q_{i}: 1 \leq i \leq l\right\} \geq 1$ or $C\left\{s_{i}: 1 \leq i \leq l\right\} \geq 1$.

(b) Let $\beta=\frac{1}{\mu}\left(\alpha_{1}+\cdots+\alpha_{l}\right)$ for some $0<\mu \leq 1$, and define $\alpha_{l+1}=-\beta$ and $\lambda_{l+1}=0$. Use the sets $\left\{\alpha_{i}: 1 \leq i \leq l+1\right\}$ and $\left\{\lambda_{i}: 1 \leq i \leq l+1\right\}$ to obtain sets $\left\{q_{i}: 1 \leq i \leq l+1\right\}$ and $\left\{s_{i}: 1 \leq i \leq l+1\right\}$ as in Lemma 4.1. Then there exists $\bar{t} \in \mathbb{R}^{+}$such that $f(\bar{t})=\beta$ only if $C\left\{q_{i}: 1 \leq i \leq l+1\right\} \geq 1$ or $C\left\{s_{i}: 1 \leq i \leq l+1\right\} \geq 1$.

Proof: These follow straightforwardly from Lemma 4.1. In each case, if neither of the conditions hold, then the relevant function has no root in $\mathbb{R}^{+}$.

Remark 4.1 The conditions given here are necessary, and in some cases also sufficient, for the existence of $\bar{t} \in \mathbb{R}^{+}$that corresponds to overshoot, undershoot or non-monotonic behaviour. Negating these conditions, we obtain conditions that are sufficient, and in some cases also necessary, to ensure that overshoot, undershoot or non-monotonic behavior do not occur. It is worth noting the conditions given depend only the coefficients $\left\{\alpha_{i}\right\}$ and the modes $\left\{\lambda_{i}\right\}$, and do not require simulating the system response.

\section{Nonovershooting and nonundershooting feed- back controllers}

In this section we see how the feedback design method described in Section 3 can be combined with the technical lemmas in Section 4 to give a systematic procedure for searching for a nonovershooting and nonundershooting feedback controller. We also discuss of the effectiveness of the search procedure.

\subsection{Gain Matrix Search Algorithm}

We assume an LTI square system $\Sigma$ in the form (1), together with a specified initial equilibrium $\left(u_{o}, x_{0}, y_{0}\right)$ and step reference $r$. The following algorithm seeks to obtain a suitable set of closed loop eigenvalues $\mathcal{L}$ and corresponding eigenvectors $\mathcal{V}$ to which the Moore's algorithm may be applied to obtain a state feedback gain matrix $F$ that yields a closed-loop step response that is neither overshooting nor undershooting.

\section{Algorithm 5.1}

(i) Begin by determining $z_{\min }$ and then solving (15) for $l$ and $q$.

(ii) For a given initial condition $x_{0}$ and reference $r$, determine $x_{s s}$ and $u_{s s}$ from (2)-(3) and hence also $\xi_{0}=x_{0}-x_{s s}$.

(iii) Choose a desired interval $[a, b]$ of the real line (where $a<b<0$ ), and form a candidate set $\mathcal{L}$ of $n$ distinct closed-loop eigenvalues containing the $n-l p+q$ minimum phase zeros of $\Sigma$, and $p$ sets of $l$ eigenvalues chosen from within $[a, b]$. 
(iv) For the appropriate value of $l$, determine the target set $\mathcal{S}$ from (16), modified according to Remark 3.2 if $q>0$. Then solve for the corresponding $\mathcal{V}$ and $\mathcal{W}$ in (7) and check if $\mathcal{V}$ is linearly independent. If it is not then return to Step (iii) and choose an alternative set of eigenvalues within $[a, b]$.

(v) Obtain the coordinate vector $\alpha$ in (17), and hence obtain the components $\epsilon_{k}$ of the tracking error $\epsilon$ from (18), for each $k \in\{1, \ldots, p\}$.

(vi) For strictly proper systems, proceed directly to Step (vii). For bi-proper systems, solve $\epsilon_{k}(0)=\mu_{k} \epsilon_{0, k}$ for $\mu_{k} \in \mathbb{R}$, for each $k \in\{1, \ldots, p\}$.

(a) For a step response without instantaneous overshoot, check $\mu_{k}>0$ for each $k \in\{1, \ldots, p\}$.

(b) For a step response without instantaneous undershoot, check $\mu_{k}<1$ for each $k \in\{1, \ldots, p\}$.

If not return to Step (iii).

(vii) If $l=1$, proceed directly to Step (viii). For $l \geq 2$, do the following for each $k \in\{1, \ldots, p\}:$

(a) For a nonovershooting response, test each $\epsilon_{k}$ for the conditions in Lemma 4.2 (if $2 \leq l \leq 3$ ), or Lemma 4.5(a) (if $l \geq 4$ ).

(b) For a nonundershooting response, test each $\epsilon_{k}$ for the conditions in Lemma 4.3 (if $l=2$ ), Lemma 4.4 (if $l=3$ ) or Lemma 4.5(b) (if $l \geq 4$ ) respectively.

(c) For a monotonic response, test each $\dot{\epsilon}_{k}$ for the conditions in Lemma 4.2 (if $2 \leq l \leq 3$ ), or Lemma 4.5(a) (if $l \geq 4$ ).

In each case, if none of the conditions in the respective lemmas are satisfied for any $k \in\{1, \ldots, p\}$, then $\mathcal{L}$ and $\mathcal{V}$ are satisfactory. If not, then return to Step (iii).

(viii) Apply Moore's algorithm to this $\mathcal{L}, \mathcal{V}$ and $\mathcal{W}$ to obtain the feedback matrix $F$ for $u$ in (4).

According to Theorems 3.1 and 3.2, the components of the closed-loop tracking error vector $\epsilon$ have the form of (14) or (18), respectively. The tests in Step (vii) ensure the coefficients $\alpha_{k, l}$ are such that overshoot and undershoot do not occur in any of the output components. For the case $l=1$, the response is globally nonovershooting and nonundershooting.

The conditions on overshoot and undershoot are independent of one another. Thus if only avoiding overshooting is important, we may perform only Steps (vi)a and (vii)a; alternatively we perform only Steps (vi)b and (vii)b to avoid undershoot only. If a monotonic response is desired, we perform Steps (vi) and (vii)c; these of course also guarantee no overshoot or undershoot. Finally note that as Lemmas 4.4 and 4.5 only offer sufficient conditions for the avoidance of overshoot, or undershoot, or extrema, using them in Step (vii) will reject some $\mathcal{L}$ that do in fact yield suitable $F$ for a nonundershooting, or nonovershooting, or monotonic, response. 


\subsection{Extensions to Non-Square Systems}

The above analysis assumed $\Sigma$ to be a square system. The eigenstructure assignment method given in [19] also considered non-square systems, and we briefly summarise the results here. For the case $p<m$, (more inputs than outputs), [19] gave conditions under which the additional control inputs could be exploited to achieve a globally nonovershooting and nonundershooting step response with any desired convergence rate (settling time). This involved augmenting $\Sigma$ with a fictitious output $y_{p+1}$. Then $p$ modes were allocated to the first $p$ components of the tracking error $\epsilon(t)$, while the remaining $n-p$ modes were "dumped" into the $(p+1)$-th component $\epsilon_{p+1}(t)$ of the tracking error. This meant that only a single exponential mode appears in each component of the tracking error, yielding no overshoot. We note here that such methods also yield no undershoot.

Secondly, for the case $p>m, \Sigma$ is not right invertible, and some targets $r \in \mathbb{R}^{p}$ are not trackable, as (2)-(3) may not be solvable. This implies that, due to the deficit of inputs, it is not possible in general to achieve a nonovershooting response for some targets. For those targets $r$ for which (2)-(3) do have solutions, and hence tracking can be achieved, [19] gave conditions under which the tracking error would could be made to contain only a single exponential mode in $m-1$ output components, and thus non-overshooting. Again, we note here that such a response would also be nonundershooting in those outputs.

In [20] the authors considered the implementation and performance of the design method when implemented with dynamic output feedback based on a Luenberger observer scheme. It was shown that state feedback tracking control scheme could also be successfully implemented with a dynamic output feedback scheme, provided the initial state estimation error is sufficiently small.

\subsection{Effectiveness of the Algorithm}

The mathematical tests employed by Algorithm 5.1 to find suitable sets of closed loop poles are computationally very tractable within MATLAB ${ }^{\circledR}$, allowing for large numbers of candidate sets of poles to be tested in an efficient manner. Very recently the authors have developed a public domain MATLAB ${ }^{\circledR}$ toolbox, known as NOUS [18], to implement Algorithm 5.1. The algorithm searches for suitable $\mathcal{L}$ and $\mathcal{V}$ to yield the required gain matrix $F$. However, there can be no guarantee that suitable $\mathcal{L}$ and $\mathcal{V}$ can always be found, for any given $\left(u_{0}, x_{0}, y_{0}\right)$ and $r$, even if $[a, b]=(-\infty, 0)$ is chosen at Step (iii). For SISO systems with real nonminimum phase zeros, there are situations in which overshoot, undershoot and local extrema are known to be unavoidable features of the step response. This is true both for state feedback and output dynamic feedback control architectures.

The general question of identifying structural a priori conditions in terms of the problem data for which the algorithm will be successful is a matter for ongoing research. The toolbox has already revealed some insights into the circumstances under which the algorithm is likely to be successful. The search algorithm is generally more effective for MIMO systems than for SISO systems, as increasing the number of outputs $p$ reduces the value 
of $l$, meaning that fewer modes need to be channeled into each component of the output. This increased effectiveness may be explained by recalling that we have assumed $\Sigma$ has at least as many inputs as outputs, since this is necessary to solve the tracking problem for an arbitrary step reference. Hence increasing $p$ also means increasing $m$, and thus we have more control inputs with which to determine the eigenstructure and hence shape the transient response. For non-square systems with $m>p$, it is generically possible to obtain a globally monotonic response, i.e. the same gain matrix renders the response monotonic in all output components, from all initial conditions, and with respect to any step reference.

\section{Examples}

In this section we consider a number of results for SISO systems describing how real nonminimum phase zeros shape the transient response, and then consider their generalization to MIMO systems. Firstly we note the classic result by Middleton on real NMP zeros and undershoot:

Theorem 6.1 [15] Let $\Sigma$ be an LTI asymptotically stable strictly proper SISO system with at least one real NMP zero. Then the step response must exhibit undershoot.

El-Khoury et al linked the number of local extrema in the step response of a SISO system to the number of system zeros:

Theorem 6.2 [6] For an asymptotically stable, strictly proper SISO system with only real poles and real zeros, the number of extrema in the step response (not including $t=0$ ) is greater than or equal to the number of zeros to the right of the right-most pole.

In [7] a simple proof was given to show that a SISO system with a NMP real zero, the output must have a zero crossing (crossing of the time axis). More generally, Leon de la Barra gave

Theorem 6.3 [11] For an asymptotically stable, strictly proper SISO system with only real poles and real zeros, the number of zero crossings is equal to the number of positive zeros.

The following example shows that none of these results generalize straightforwardly to MIMO systems.

Example 6.1 Consider the strictly proper MIMO system $\Sigma_{1}$ with

$$
A=\left[\begin{array}{cccc}
0 & 0 & -3 & 0 \\
0 & 0 & 0 & 4 \\
0 & 6 & -10 & 0 \\
0 & -10 & 0 & 0
\end{array}\right], B=\left[\begin{array}{cc}
-5 & -5 \\
-5 & 0 \\
0 & -2 \\
0 & 1
\end{array}\right], C=\left[\begin{array}{cccc}
-4 & 0 & -5 & 0 \\
-4 & 0 & -4 & 0
\end{array}\right], D=\left[\begin{array}{ll}
0 & 0 \\
0 & 0
\end{array}\right] \text {. }
$$


The system has two real NMP zeros at 12.8151 and 2.1849. We assume zero initial conditions and a step reference of $[1,1]^{T}$. Using Algorithm 5.1, we sought a gain matrix $F$ to deliver a monotonic response. The search was successful, yielding

$$
F=\left[\begin{array}{rrrr}
-6.11 & 23.14 & 6.16 & -25.37 \\
9.24 & -15.62 & -0.75 & 18.84
\end{array}\right] \text {, }
$$

Applying the control law (4), with this $F$ and with $u_{s s}$ and $x_{s s}$ obtained from (2)-(3), yields a transient responses as shown in Figure 3. We see the step response is monotonic in both outputs. The gain matrix $F$ places the closed-loop poles at $\{-41,-40,-35,-5\}$ and thus the closed-loop system is stable and strictly proper with only real poles and zeros. This example shows that for MIMO systems, the presence of real NMP system zeros does not necessarily imply the outputs must exhibit undershoot, local extrema or zero crossings.
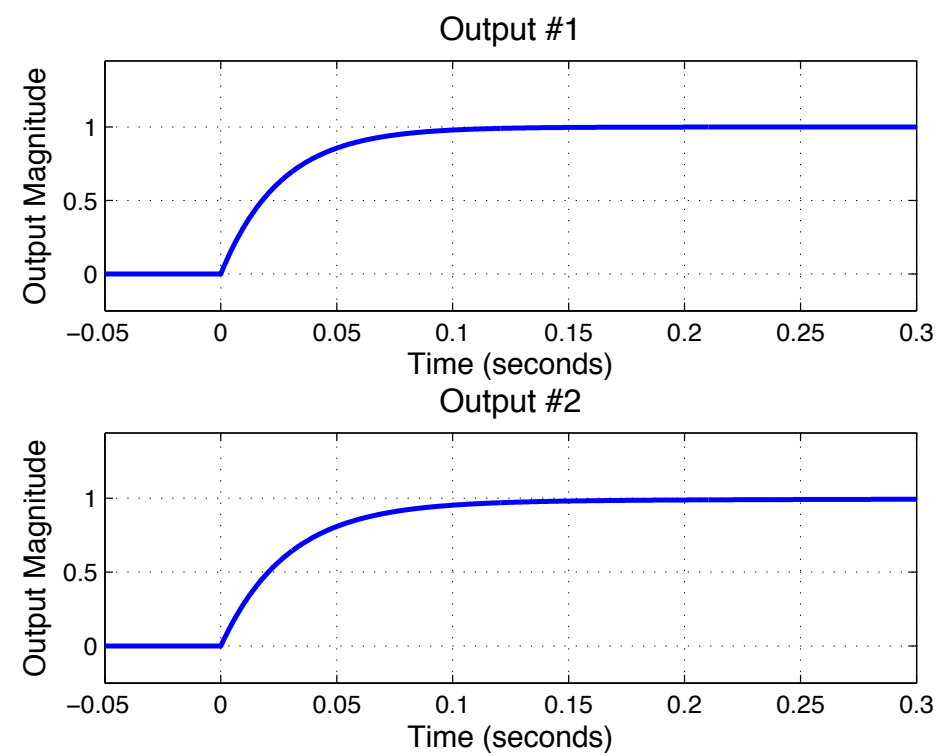

Figure 3: Output components for $\Sigma_{1}$.

Example 6.2 Here we consider the non-square MIMO system $\Sigma_{2}$ with

$$
\begin{aligned}
& A=\left[\begin{array}{ccccc}
-6 & 0 & 0 & 0 & 0 \\
3 & 3 & 0 & 0 & 0 \\
0 & 0 & 2 & 0 & 2 \\
-1 & 0 & 2 & 0 & 0 \\
-2 & 0 & 0 & 0 & 2
\end{array}\right], B=\left[\begin{array}{cccc}
0 & 0 & 0 & 0 \\
0 & 0 & 0 & -3 \\
0 & 4 & 2 & 0 \\
1 & -1 & 0 & -1 \\
0 & -1 & 0 & 0
\end{array}\right], \\
& C=\left[\begin{array}{ccccc}
-1 & 0 & 0 & 0 & 0 \\
3 & 0 & 0 & 0 & 9 \\
1 & 0 & 0 & 0 & 0
\end{array}\right], D=\left[\begin{array}{cccc}
0 & 0 & -2 & 0 \\
0 & 3 & -3 & -3 \\
0 & 0 & 2 & -2
\end{array}\right] .
\end{aligned}
$$


The system has four real zeros at $\{-6,2,3,5\}$. Using our adaptation of the control design method to non-square systems, we sought a gain matrix $F$ to deliver a globally monotonic response. The search was successful, yielding

$$
F=\left[\begin{array}{ccccc}
68419 / 8250 & 802 / 125 & -1121 / 125 & -6 & -1639 / 250 \\
-5351 / 2475 & -16 / 75 & 6 / 25 & 0 & 127 / 25 \\
5537 / 4950 & -12 / 225 & -36 / 25 & 0 & -162 / 25 \\
4 / 9 & 4 / 3 & 0 & 0 & 0
\end{array}\right],
$$

This $F$ places the closed loop poles at $\{-6,-6,-2,-1,-1\}$. We simulated the transient response for three sets of initial equilibrium conditions $x_{0 i}$ and targets $r_{i}$ as follows:

$$
\left(x_{01}, r_{1}\right)=\left(\begin{array}{cc}
-1.039 & 10 \\
1.118 & 15 \\
-1.261 & 20 \\
-0.660 & * \\
0.068 & *
\end{array}\right),\left(x_{02}, r_{2}\right)=\left(\begin{array}{cc}
-1.610 & 5 \\
1.338 & 0 \\
-1.778 & -5 \\
1.422 & * \\
-0.239 & *
\end{array}\right),\left(x_{03}, r_{3}\right)=\left(\begin{array}{cc}
-1.610 & -10 \\
1.338 & -15 \\
-1.778 & -20 \\
1.422 & * \\
-0.239 & *
\end{array}\right)
$$

Applying the control law (4), with this $F$ and with $u_{s s}$ and $x_{s s}$ obtained from (2)-(3) for each target $r_{i}$, yields sets of transient response curves as shown in Figure 4. For $t>0$, the response is monotonic in all outputs, for each set of initial equilibrium conditions and targets. Note however that the responses may exhibit initial undershoot or overshoot from some of these initial conditions, due to the presence of the $D$ term in this system.

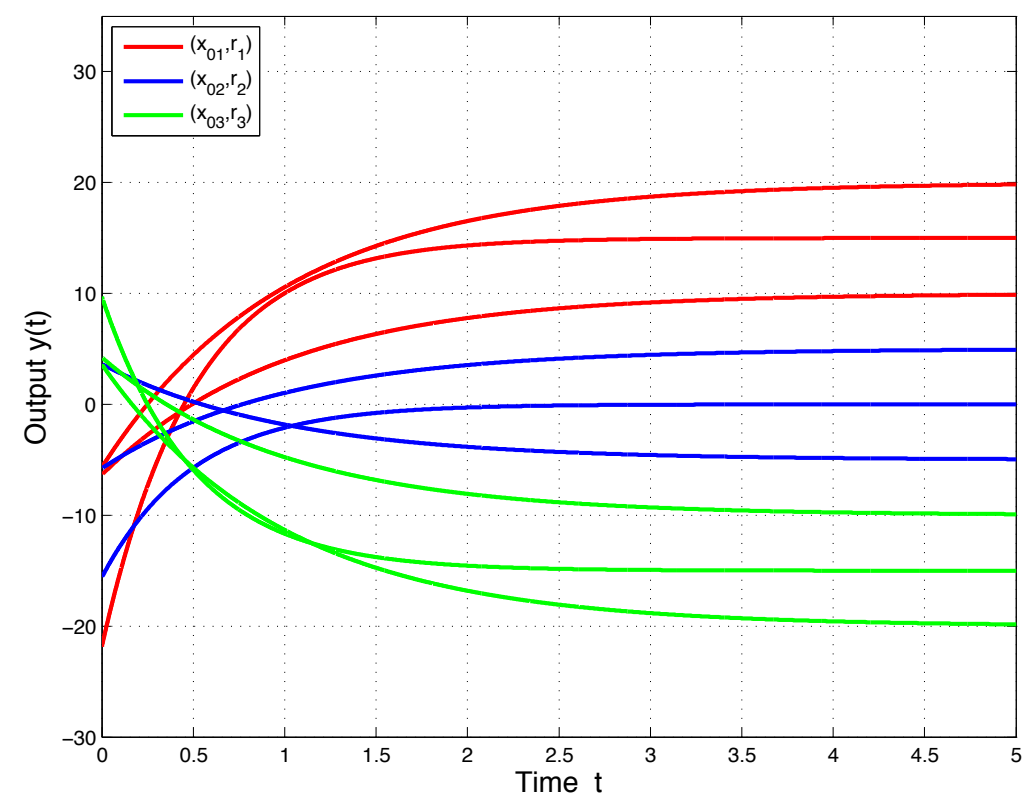

Figure 4: Output components for $\Sigma_{2}$. 


\section{Acknowledgements}

The authors would like to thank Julio Braslavsky for some helpful suggestions on the definition of undershoot for bi-proper systems. We would also like to thank the anonymous reviewers for their helpful suggestions.

\section{Conclusion}

We have extended the design method for a linear state feedback tracking controller to achieve a nonovershooting step response for suitable MIMO systems given in [19]. Conditions are given under which a suitable gain matrix may be obtained to render the step response nonovershooting, or nonundershooting, or both. The method is described here for continuous-time square systems, and is applicable to both strictly proper and bi-proper systems, which may be of minimum or nonminimum phase. The method may also be applied to discrete-time systems, and also non-square systems. We refer the reader to [19] for the details of those cases. In each case the transient response is shaped by a suitable application of Moore's algorithm. The method avoids the inherent conservatism of designing for a sign-invariant impulse response, since this is not essential to avoid undershoot and overshoot in the step response. To the best of the authors' knowledge, this is the first linear control scheme that can simultaneously provide both a nonovershooting and nonundershooting step response for a MIMO system. Moreover, it is the first method (MIMO or SISO) for avoiding undershoot that does not require monotonicity of the step response.

\section{References}

[1] B.D.O. Anderson, M. Deistler, L. Farino and L. Benvenuti, Negative realization of a linear system with nonnegative impulse response, IEEE Transactions on Circuits and Systems-I: Fundamental Theory and applications, vol. 43(2), pp. 134-142, 1996.

[2] M. Bement and S. Jayasuriya, Use of state feedback to achieve a nonovershooting step response for a class of nonminimum phase systems, Journal of Dynamic Systems, Measurement and Control, vol. 126, pp. 657-660, 2004.

[3] S. Darbha, On the synthesis of controllers for continuous time LTI systems that achieve a non-negative impulse response, Automatica, vol. 39, pp. 159-165, 2003.

[4] S. Darbha, and S.P. Bhattacharyya, On the synthesis of controllers for a nonovershooting step response, IEEE Transactions on Automatic Control, vol. 48(5), pp. 797-799, 2003.

[5] S. Darbha, and S.P. Bhattacharyya, Controller synthesis for sign invariant impulse response, IEEE Transactions on Automatic Control, vol. 47(8), pp. 1346-1351, 2002. 
[6] M. El-Khoury, O.D. Crisalle, and R. Longchamp, Influence of zero locations on the number of step-response extrema, Automatica, vol. 29, pp. 1571-1574, 1993.

[7] J.B. Hoagg and D.S. Bernstein, Nonminimum-Phase Zeros, IEEE Control Systems Magazine, pp. 45-57, 2007.

[8] K. H. Johansson, Interaction bounds in multivariable control systems, Automatica, Vol 38, pp. 1045-1051, 2002.

[9] J. Kautsky, J. N.K. Nichols and P. Van Dooren, Robust Pole Assignment in Linear State Feedback, International Journal of Control., vol. 41, pp. 1129-1155, 1985.

[10] E. Laguerre, On the Theory of Numeric Equations, Journal de Mathématiques pures et appliquées, $3^{e}$ série, t. IX, 1883. Translated by Stewart A. Levin, available from http://sepwww.stanford.edu/oldsep/stew/laguerre.pdf

[11] B.A. Leon de la Barra, Sufficient conditions for monotonic discrete time step response, Journal of Dynamic Systems, Measurement and Control, vol. 116, pp. 810814, 1994.

[12] B.A. Leon de la Barra, On undershoot in SISO systems, IEEE Transactions on Automatic Control, vol. 39, pp. 578-581, 1994.

[13] K. Lau, R.H. Middleton and J.H. Braslavsky, Undershoot and Settling Time Tradeoffs for Nonminimum phase systems, IEEE Transactions on Automatic Control, vol. 48, pp. 1389-1393, 2003.

[14] Lin, S.K and C.J. Fang, Nonovershooting and Monotone Nondecreasing Step Responses of a Third-Order SISO Linear System IEEE Transactions on Automatic Control, vol. 42(9), pp. 1299-1303, 1997.

[15] R.H. Middleton, Trade-offs in linear control system design, Automatica, vol 27, 1991, pp. 281-292.

[16] B.C. Moore, On the Flexibility Offered by State Feedback in Multivariable systems Beyond Closed Loop Eigenvalue Assignment, IEEE Transactions on Automatic Control, vol. 21(5), pp. 689-692, 1976.

[17] J. Maciejowski, Multivariable Feedback Design, Addison-Wesley, 1989.

[18] A. Pandey and R. Schmid, NOUS: a MATLAB ${ }^{\circledR}$ toolbox for the design of nonovershooting and nonundershooting multivariable tracking controllers, available from http://people.eng.unimelb.edu.au/rschmid/documents/NOUS1.0.zip

[19] R. Schmid and L. Ntogramatzidis, A unified method for the design of nonovershooting linear multivariable state-feedback tracking controllers, Automatica, vol. 46, pp. 312-321, 2010.

[20] R. Schmid, and L. Ntogramatzidis, Achieving a nonovershooting transient response with multivariable dynamic output feedback tracking controllers, Proceedings of the 48th IEEE Conference on Decision and Control (CDC 2009), Shanghai, 2009. 
[21] J. Stewart and D.E. Davison, On Overshoot and Nonminimum phase Zeros, IEEE Transactions on Automatic Control, vol. 51(8), pp. 1378-1382, 2006. 